\title{
Transparently programming heterogeneous distributed systems
}

\author{
T. Wolff \\ K.-P. Löhr \\ Institut für Informatik, Freie Universität Berlin \\ Takustraße 9, D-14195 Berlin \\ \{lohr,wolf\}@inf.fu-berlin.de
}

\begin{abstract}
The Heron system is a language-independent platform for distributed object systems. As opposed to similar platforms such as Amadeus or CORBA, it neither meddles with the language systems nor compromises distribution transparency. This paper, after presenting the basic design issues, focuses on the notion of remote inheritance and on how to support language heterogeneity without writing IDL code.
\end{abstract}

\section{Keywords}

Distributed object systems, remote inheritance, heterogeneity, distribution transparency.

\section{INTRODUCTION}

The integration of distribution and object orientation has been paid much attention by both researchers and practitioners in recent years. It has been commonly accepted that there is no unique, best way to combine the two. Distributed object systems come in different flavours and different degrees of integration, language dependency etc. In particular, they differ in their approach to accommodating heterogeneity, both on the systems and the languages level.

One problem of distributed and heterogeneous systems is that distribution and heterogeneity tend to remain visible in the application code, thus burdening the software with error-prone and unproductive overhead. Software engineers who just want to produce distributed applications using their favourite language(s) have come to accept as a fact of life that distribution cannot be fully transparent. On the other hand, academic developments often come up with new languages or language features that achieve some transparency but are weakly supported and not accepted by users. 
The Heron project takes an intermediate way without being a compromise. It adds the option of distribution to existing programming environments without changing their basic properties. It offers a support platform that is transparent to the software with respect to distribution, system architecture, and programming language. Furthermore, it is also transparent to the underlying local language systems so that basically any compilation system for an object-oriented language can be employed as part of a Heron system.

Why should distribution be transparent at all? For most of the various kinds of software intended to run on a distributed system, the details of distribution do not have to, and thus should not, be apparent in the program. This is especially true for the use of distribution for increase of throughput, but may still be valid even if distribution is employed to make use of specific local hardware or other resources.

HeRon distributes objects transparently. Furthermore, it even splits objects transparently, yielding a finer unit of distribution than objects.

Possible heterogeneity and the idea of using public server objects of open systems in a more language-integrated way require solutions to be most general with respect to conceivable language and program structures. With this in mind, we have constructed a system that also supports multi-language programming transparently on the object level.

Previous publications cover several aspects of the project: [FINKE ET AL 1993] describes the basic design ideas of Heron, [Löhr Piens Wolff 1994] gives some popular motivation of the importance of transparency in distributed systems, [PIENS 1993] covers the configuration system within Heron, [WoLfF 1995] describes implementation principles and technical details of the transparent distribution interface, and [HOFFMANN WEINBERGER WOLFF 1995] presents technical implementation concepts of the heterogeneous extension of HERON.

This paper focuses on the platform character of HERON, aiming at its suitability for easy transparent handling of heterogeneous distributed systems for software development.

\section{I.I Classification of approaches}

Many concepts and systems for distribution of object-oriented languages have been developed. They can be classified into 6 different approaches:

\section{Language-oriented approaches}

Distribution is integrated into a programming language.

- Distributed language: A new dedicated programming language is designed to support distribution from the beginning. Thus, the language can enjoy specific semantic properties that take into account distribution in a clean way (e.g. SR [ANDREws OLsSON 1993], Emerald [RAJ ET AL 1991]).

- Language extension / modification: An existing programming language is extended by distribution elements; this often includes modified semantics, especially concerning parameter passing. Examples are: Distributed Eiffel ([Gunaseelan LeBlanC 1992]), Distributed Smalltalk ([BENNET 1990]).

\section{Language-transparent approaches}

A method is developed to distribute programs of an existing programming language without need for special programming and without modified semantics.

- Language transparency by dedicated implementation: Distribution is supported by a specialized compiler or by making extensive use of specific properties of a given runtime system (e.g. Eiffel** 
[McHugh CaHill 1993]). This is what a company (e.g. a compiler vendor) would probably do in order to offer their customers additional flexibility in using distribution.

- Full transparency in all aspects: The implementation is achieved by additional or substitutional techniques alone, on the given language level and without relying on language implementation details (our approach, in the Heron project).

\section{Library approaches}

Without modification of given language systems, libraries are designed to enable explicit programming of distribution.

- Distribution library: A library supports programming of distribution on the usual language level, e.g. sending objects to a receiver on a remote machine. Examples: the announced EIFfeLNet library by ISE (Eiffel vendor); DCE ([OSF 1993]) as a system specifically tailored to the requirements of the $\mathrm{C}$ language.

- Communications library: A runtime library supports message exchange among distributed parts of a program, requiring explicit handling of elementary data and taking care of the relationships between the program parts (e.g. PVM [SUNDERAM 1990]; DCE in its organizational aspects).

\section{I.2 The Heron approach: transparency in all aspects}

A speciality of the HERON project is the realization of transparency in three aspects:

- Syntactic transparency: The presence of distribution shall affect the syntax of a program: remote operation calls shall look the same as local ones. Necessary means of determining the residence of new objects shall be placed outside of the program. In conjunction with this, Heron allows for separate configuration descriptions ([PIENS 1993]).

Library systems require distribution aspects to be made explicit. They complicate the construction of programs in an error-prone way. This is a wrong approach from a systematic point of view.

- Semantic transparency: Remote objects and invoked operations shall behave as if they were local. This is especially difficult with parameter passing semantics but also with operations combining distributed object values, like copying and comparing. Other examples are loosely-defined language constructs like arrays in Eiffel whose range of contents (descriptor only or element values) is not quite clear, or pointers in the $\mathrm{C}$ languages which may always refer to arbitrary and unknown quantities of elements.

- Language system transparency: We do not write a new compiler nor is our distribution system in any way connected to the internals of an existing compiler and runtime system for the nondistributed language. Our aim is to demonstrate what can be achieved if a language-conforming environment attached to a closed, possibly proprietary compilation system is the only way of implementation available. (The relevance of this approach is often not obvious nowadays because systems for the $\mathrm{C}$ language can usually make use of common knowledge of uniform implementation internals, e.g., of parameter storage.) Especially if a less wide-spread high-level language is used, it is certainly a good advice not to depend on the internals of one specific compilation system. Moreover, as we also strive to accommodate language heterogeneity, we cannot rely on compatibility of compiler internals. 


\section{I.3 Object-oriented language model}

We have chosen Eiffel ([MEYER 1992], [MONNINGER 1993]) as the primary reference language for Heron. The aspects of inheritance that are of special interest for the project, namely all conceivable relations between subclasses and superclasses and all their modifying properties, are completely and most easily represented by Eiffel. The complete set of structuring features is especially important to support language-independent system design and thus enable the heterogeneous extension.

\section{IMPLEMENTATION PRINCIPLES}

The central functionality of object interaction, the operation invocation, is turned into remote object invocation when the two affected objects reside on two different nodes of a network (virtual nodes, to be precise, to accommodate the special case of independent address spaces on one machine). Remote object invocation must be performed in a completely transparent fashion: syntax and semantics must be those of a local invocation. No syntax or semantics change should be accepted to enable the remote object invocation. The classical method to achieve this in a module context (RPC) are stub modules ([BIRREL NeLSON 1984]). In analogy to those, we use representative objects of two kinds: proxy objects which serve as representatives for called objects and driver objects acting on behalf of the callers. These auxiliary objects organize the transformation between language-conforming operation calls and explicit operation request messages. They invoke the actual communication using a distribution runtime system (i.e., a runtime system managing distribution, DRTS). For an illustration, see FIGURE I. The DRTS module is only a small interface to the underlying communication system employed for passing messages between the representative objects. In our current implementation, we use PVM ([SUNDERAM 1990]) which is an adequate and versatile communication system for this purpose, interfacing just at the appropriate level.

\section{I Implementation techniques}

The transparent integration of the representative object technique into the local language compilation systems works by substituting a proxy class for the respective original class on any machine that uses objects of that class remotely, and installing a driver class on any machine on which objects of the respective class may reside and be used remotely. This substitution solution is the optimal system assembly approach in Heron although for some exotic situations in distributing objects a transformation solution must be employed which also uses some modified version of the distributed class or even the actual application program. However, this is still transparent and feasible.

\subsection{Basic principles of parameter handling}

In consequence of an operation call, parameter objects (of class B in Figure I) may be referenced by the called object and respective invocations have to be led transparently to the original parameter object which still resides on the machine of the caller (or even a third machine). Therefore, representative objects of similar functionality have to be created and installed on every remote object invocation for every parameter of object reference type. The functionality they provide is also known as 'back-call' in the simpler case of dedicated client and server modules. 


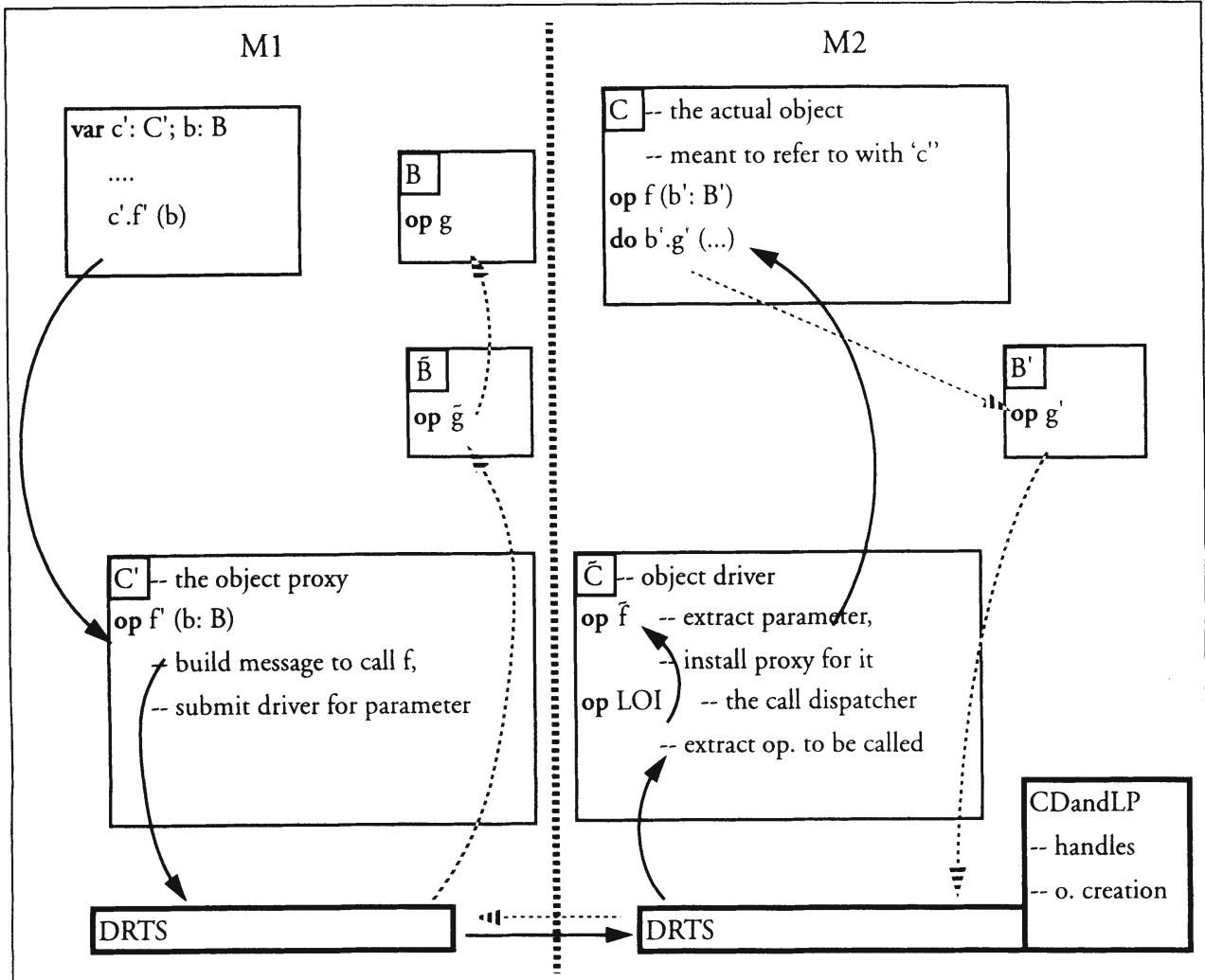

Figure I. For every class $\mathrm{C}$ of the original program, $\mathrm{C}^{\prime}$ is its proxy class and c' a variable of it; $\bar{C}$ is its driver class. Operation names are marked accordingly. (While the real name of driver classes does not matter, the proxy classes, acting as substitutes, must actually bear the same real name as their respective original classes. So the name annotation ' is only understood as a hint to indicate which sort of class is displayed.)

EXPLANATIONS: LOI: local object invocation. DRTS: distribution runtime system. CDandLP: create distributor and local propagator.

$\longrightarrow$ : an operation call on its way from the invoker ('client' of this invocation) to the called object (in the 'server' of this invocation).

........... : an operation call on its way from the server object of the first call (now a client) to an object in the former client part of the distributed situation ('back-call').

Situation: Once the object of class $\mathrm{C}$ on $\mathrm{M} 2$ has been created, it can be invoked remotely from $\mathrm{M} 1$ on the path shown by solid arrows, first using the proxy of class $\mathrm{C}^{\prime}$ which is substituted for $\mathrm{C}$ on M1. As an actual parameter, the local object reference $b$ is conveyed to M2 where it must be incorporated into a proxy of class $\mathrm{B}^{\prime}$ in order to be used remotely. 
This is one of three possible approaches in remote passing of object reference parameters; the other two are not suitable for our approach:

- Transferring structured data (like in [HerLihy Liskov 1982]) is mainly useful for efficiently supplying remote servers with complex information. Passing values for reference parameters is not acceptable in a transparent system since the semantic property of object identity would not be maintained; this problem becomes especially (but not exclusively) apparent if concurrent computing is added to the system. (Call by value, on the other hand, is performed transparently by remote object value transfer.)

- Call by move would be a semantics-conforming alternative but is of questionable efficiency gain without explicit language provisions as suggested, e.g., in [RAJ ET AL 1991] or in [ACHAUER I993].

\subsection{Object creation and transparency}

Any remote object will first have to be created before its use (unless we address public server objects which may exist independently of a program run). The actual performance of remote object creation shall be transparent, like any object invocation, putting the object proxy's creation / initialization part in charge of invoking the creation procedure on the remote machine, and leaving the actual task of creation to a special module besides the remote DRTS, the create distributor.

Concerning remote object creation, the most important question to be realized in the concept design is 'Which machine should we put the new object on?' It may be debatable whether machine selection should also be completely transparent or whether programmers may have an interest in specifying the target machine with the creation. In Heron, the evaluation of any parameters that might affect the selection of the host machine for a new object is left to a specific configuration class which is automatically derived from the configuration description. This description of the distribution situation a program is supposed to run in is syntactically separate from the actual program.

The cooperation of the proxy object and its configuration class can be seen in Figure 2: Transparent creation of a remote object by !!c1.init $(x 1, \ldots, x n)$ first activates the proxy which calls the configuration routine for the respective initialization operation. This routine evaluates the respective configuration function $\mathcal{M}$ using both the local parameters and additional global information.

Establishing the connection of the initialization part of the representative class $\mathrm{C}^{\prime}$ and the remote location determination function $\mathcal{M}$ taken from a configuration class $\mathrm{C}^{\mathrm{conf}}$ is one of the tasks of the configurator [PIENS 1993].

\subsection{Realizing distribution and inheritance}

If class $C$ inherits from class $D$, the proxy class $C^{\prime}$ also inherits from $D^{\prime}$ and the driver class $\tilde{C}$ inherits from $\tilde{D}$. This way the operations in charge of implementing the remote object invocation for their respective operations of the original class are automatically available in the representative objects without having to reimplement their handling in every subclass representative. If we can stick with this principle throughout all special aspects of object features and inheritance hierarchies, it eases the task of generating the representative classes significantly; it also makes it more efficient since we can generate the representatives from the respective class text alone, without analysis of the superclasses.

An immediate advantage of the structure-preserving principle is that semantic relationships between names and locations of inherited object features (search rules for identifying methods, 'method dispatch' rules) are isomorphically transferred to the proxy hierarchy (FIGURE 3), no matter how 


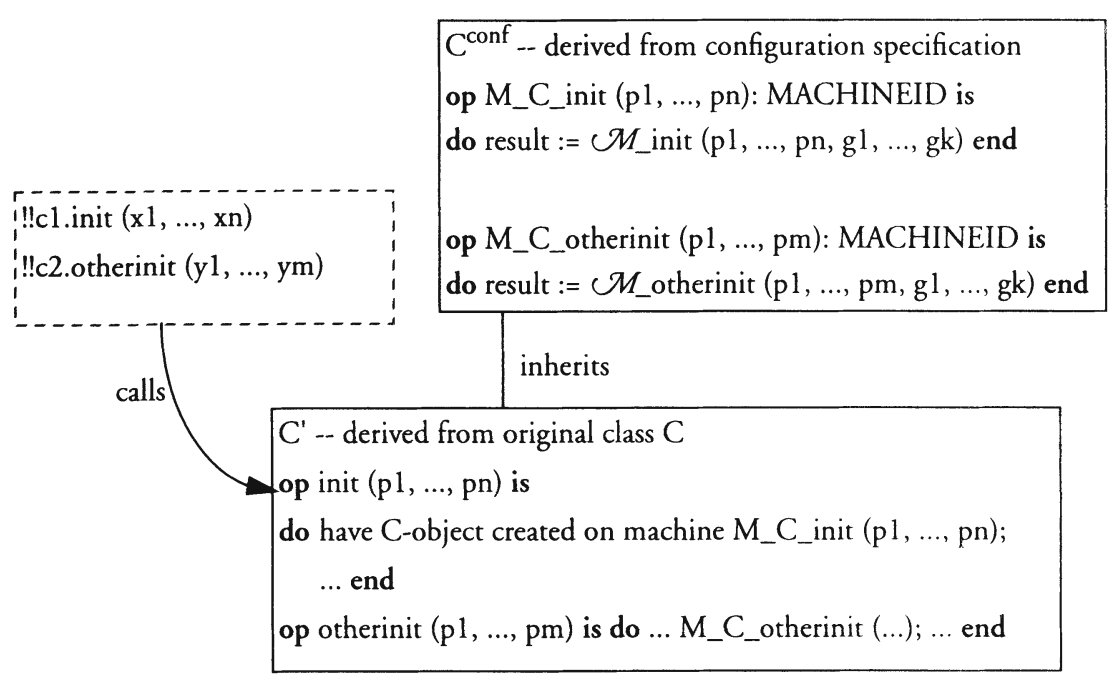

FIGURE 2. Incorporating the configuration specification into the proxy at runtime
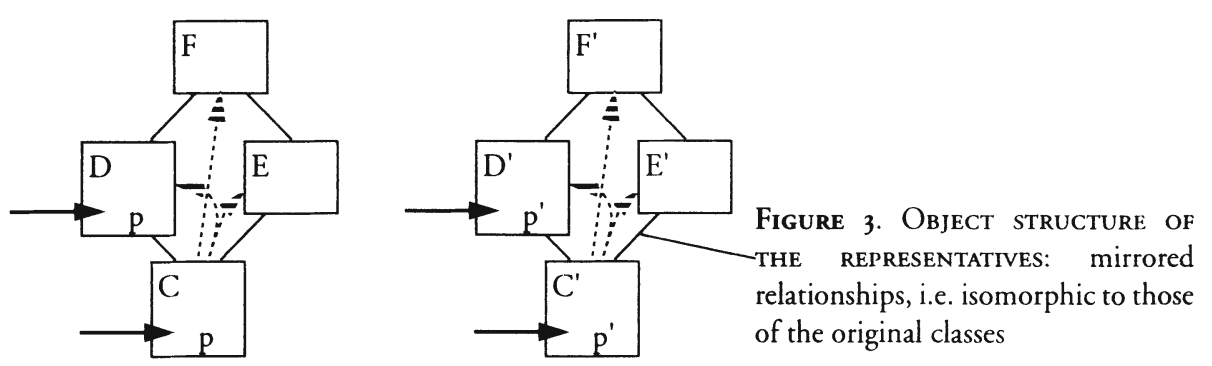

they are defined. This principle saves us some trouble with naming or scoping conflicts if different class structures were used. It also gives us some free solutions for the isomorphic mapping of redefinition or renaming relations from the caller to the server of this distributed situation.

\section{REMOTE INHERITANCE AND SPLIT OBJECTS}

Some special application cases might call for intra-object distribution. Let us assume that different parts of the code of a class hierarchy need access to resources located on different machines (such as special hardware or computing power). In order to maintain our claim to maximal transparency, we supply a concept and transparent implementation of split objects. This enables the programmers to use special remote features by still inheriting from the classes handling them, and, again, between different languages as well.

By being even finer-grained in distribution than the most obvious unit of distribution, the object, we meet more sophisticated implementation problems. Immediately the question arises how mutual access between distributed parts of an object can be organized. For both systematic and imple- 
mentation aspects, it seems reasonable to restrict remote object parts to some unit of modularity which is already present in the system. We set up support for a concept such that parts of objects corresponding to their respective superclasses can reside on different machines, thus establishing remote inheritance - or rather distributed inheritance, named from the global view.

The transparent implementation of distributed inheritance requires two additional implementation schemes, the partial proxies and the split object setup procedure.

We do not only need representatives for remote objects; rather, we must combine object part representatives together with local object parts into the local implementation class hierarchies for split objects. We call them partial proxies in contrast to the monolithic proxies as described before.

Their responsibilities are:

- Handling operations calls from other parts of the same object. Operation invocations within the class hierarchy must and can be handled just like external object invocations to be directed to the appropriate remote object part. Unfortunately, assignment to an object variable cannot be handled by a partial proxy for syntactic reasons unless a transformation solution would be employed.

- Redefining and renaming entry points of superclasses. This does not only implement the corresponding functionality of the original classes as does the same situation in monolithic proxies. It can also redirect a local operation call to a remote one or a remote to a local one.

- Maintaining information about where the remote object part resides. Whereas fortunately the two aspects above do not need special mechanisms in addition to the monolithic case, the information about the object location can no longer be kept once in a proxy as before but must be maintained separately in each partial proxy.

The actual realization of the split object implementation uses one implementation object on each machine which shares part of the whole object. We call these object segments of the split object whereas an object part is that part of an object corresponding to one particular superclass of the class hierarchy. An object segment comprises one or more object parts and partial proxies for all remote object parts.

In addition to partial proxies which mainly manage the flow of computation and access within the split object, we still need driver objects for the whole object to handle remote invocations from outside of the object, one for each machine that a part of the driven object resides on. This leads to situations like the example shown in Figure 4.

Setting up control information in all parts of a split object is an especially complex problem and is described in detail in [WoLfF 1995].

\section{HETEROGENEOUS EXTENSION}

Given the implementation system of auxiliary objects, the multi-language situation looks like in Figure 5. We are facing two problems:

- How to generate (or 'cross-compile') the interfacing auxiliary classes among different languages.

- How to define the interface structure that the auxiliary classes realise between the distributed program components.

For the cross-generation system for multiple languages, instead of implementing lots of cross-generators, we use a front-end and back-end per language via an interface language. This interface lan- 


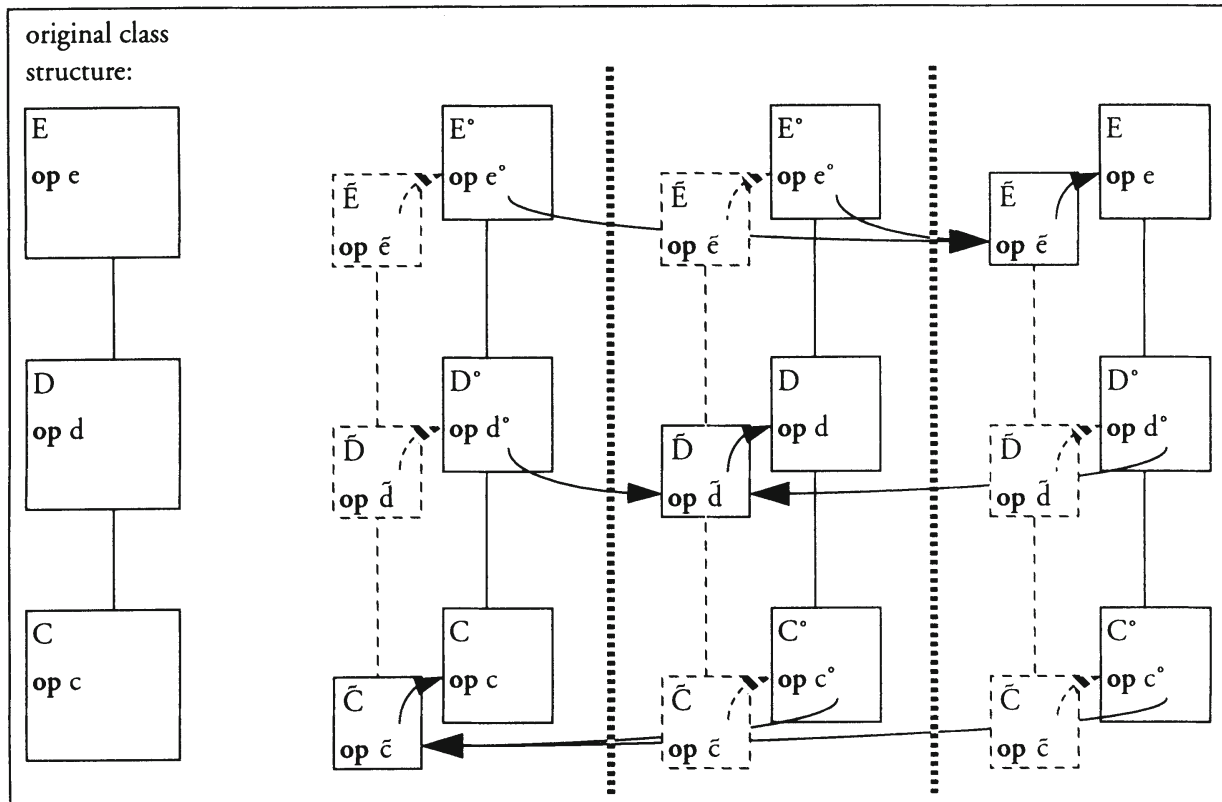

Figure 4. Split object: Object parts corresponding to the three classes C, D, E of a class hierarchy reside on three different machines. Each of these original parts is completed to the full hierarchy by partial proxies (representatives for object parts). They are denoted by a ${ }^{\circ}$ sign. All three hierarchies are accompanied by their driver object hierarchies. Those parts of them corresponding to a local original object part (depicted with a solid border) have to handle incoming remote requests from other parts of the split object or from other objects. The other parts of the driver objects (depicted with a dashed border) are only there to complete the inheritance relations of the driver class.

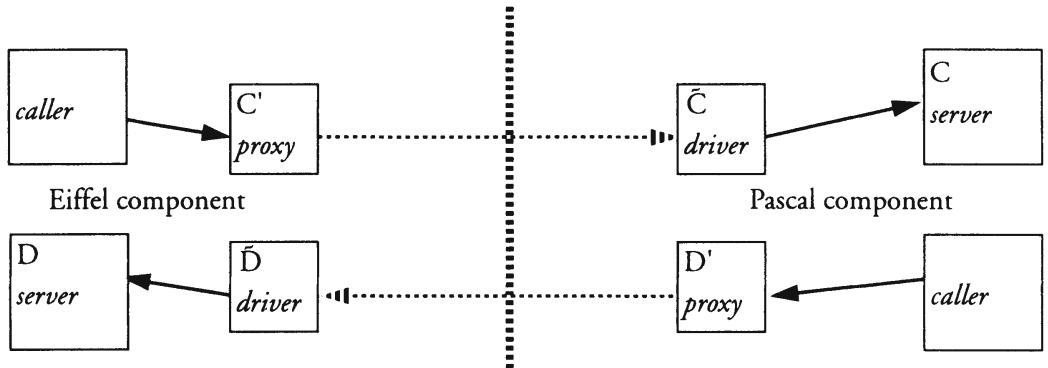

Figure 5: Heterogeneous auxiliary objects 
guage has been developed along the guidelines of the object-oriented feature set of Eiffel in order to be most powerful for its purpose.

For the first heterogeneous project extension, we have chosen Turbo-Pascal ([BoRLAND 1993]), a language well established in the PC world.

The system interface to Turbo-Pascal reveals some general problems of composing object-oriented software from different language modules:

- different access protection of object components,

- access to the objects by name/variable/pointer,

- strict object orientation versus coexistence with other (modular/data) structures,

- object destruction versus garbage collection,

- different concepts of redefinition or nested scoping, different variance requirements for subclass conformance (covariant/contravariant parameters),

- minor incompatibilities like generic classes, multiple inheritance, or constant attributes; these amount to the simple fact that in a language-heterogeneous interface only those language constructs can be used that exist in the partner language as well. This subset property is a natural restriction but it does not restrict multiple partners to be content with the intersection of all concepts since the underlying model is rather the union of concepts, thanks to Eiffel.

\section{BUILDING COMPONENTS OF A DISTRIBUTED PROGRAM}

A distributed program comprises several components on different nodes in a network. A component is a compiled and linked piece of code that is ready to be loaded into an address space.

Building components is the task of the Hekon configurator tool. A component description - primarily consisting of import/export directives referring to classes - and source code are fed into the configurator. Proxy and driver classes are then generated automatically, according to the import/export directives. When those classes and the programmer-supplied classes have been compiled, communication support code is looked up in a library and everything is linked together to form the component. Note that the local language system - as is - is employed for generating the component. - A detailed description of the configuration process and of the configuration description language is given in [PIENS 1993].

A distributed program is started by starting an instance of its root component. The program then evolves, causing private instances of remote components to be created on demand and/or accessing services of already existing public instances (i.e., servers), if this was specified in the configuration description. A name service supports the identification of both components and servers.

\section{FINAL REMARKS}

\section{I Implementation}

The Heron system was first implemented for the Eiffel language. The generator was written in Eiffel and installed on Unix workstations and MSDOS PCs. For the heterogeneous extension, Borland Pascal was chosen as first other language. For communication, an easy-to-build first implementation was using PVM. But as Heron does not rely on any PVM specifics, other choices might be DCE or MPI ([MPI 1994]). 


\subsection{Related work}

Heron demonstrates that it is possible to apply a technique of merely supplementary implementation concepts to achieve full distribution transparency in object-oriented systems. As pointed out in I.I, most other approaches to programming distributed systems are placed on one of two different levels. There are distributed language extensions and distributed languages, where the latter will usually present less problems to both users and implementors than the former since a given distribution semantics can be followed in a uniform compilation environment. Distribution platforms such as DCE, PVM, and even Corba ([OMG 1991]) are only the lowest level of implementation from the application programmers' point of view. Their presence in programs in the form of an interface, even if the more advanced of those systems are used, is not considered desirable for systematic construction of distributed application programs. It should also be noted that the style of programming they require is more like sets of communicating program fragments than uniform distributed programs.

Interface specification aids have been developed (e.g. IDL of NCS [JoHNSON LEACH 1987], later DCE, and CORBA; also Matchmaker [Jones RASHID 1986]); they are mainly useful in heterogeneous environments but they are also required for languages with poor type systems like the single-letter one where parameter types could not otherwise be determined properly ([WoLfF 1988]). The IDL of Corba is oriented towards $\mathrm{C}++$. Consequently, its object model is rather simple; e.g. there is no repeated inheritance.

In contrast to explicit interface languages, the intermediate language of Heron does not get into contact with the user; it is merely an implementation item for the cross-generation procedure. The configuration description of HERON does not describe interfaces either; it merely defines the association of classes with the components of the distributed program.

Avoiding the use of an explicit IDL has also been advocated in the Amadeus project ([HoRN CAHILl 199I], [McHugh CAHILl 1993]) where 'shadow interfaces' were suggested. While Amadeus was more ambitious than Heron in several respects (incorporating, e.g., persistent objects), it had to resort to meddling with the language systems for its implementation.

The Concert system also uses a hidden IDL, called Concert Signature Representation ([AuERвасн Russell 1994]). As opposed to Heron, however, Concert is not object-oriented and relies on language extensions and special compilers; it does not achieve full distribution transparency.

DC++ ([SCHILL MocK 1993]) provides more functionality with respect to language interface and transparency than the communication-oriented systems (it uses one of them, DCE). It is, however, not completely transparent, not completely compiler-independent, and also makes use of an explicit IDL specification of remote classes.

Systems that address inheritance (DC++; also [NolTe 1994]) usually have problems with it, especially in the area of redefinitions. Thanks to the implementation principles outlined in 2.4, the Heron distribution system has no problem with inheritance.

\subsection{Conclusion}

Our approach strives for establishing a completely transparent distribution system for high-level object-oriented languages by hiding distribution details from the actual programs and maintaining the semantics of the host languages.

In contrast to the currently prevailing library approaches, our work stands more in the tradition of classical RPC systems using the stub technology. Like others, we have extended that technology to the situation of object-oriented systems, but we have avoided semantic compromises. In contrast 
to stub systems, the HERON representative system has to pay special attention to various aspects of inheritance and object creation. Moreover, split objects and the lazy implementation of split object creation ([WoLfF 1995]) makes remote inheritance a unique feature of the Heron system.

The main problem of most approaches based on given languages is that they tend to not only extend, but also modify the semantics of the original language. (Especially the semantics of reference parameters is usually not respected but just smoothed out without much fuss.) The difference of our approach is its uncompromised priority of transparency with respect to both syntax and semantics. Moreover, it has a clear concept of what to do about inheritance which is rarely mentioned in connection with other approaches.

The language Eiffel (version 3) supports the implementation of Heron by providing a very general and flexible-to-use inheritance system but it also puts obstacles to our implementation. An example is the 'once function', the unspoken module concept of Eiffel; it requires complicated special treatment because it pretends to be an object feature although it is not.

As has been pointed out, the Heron approach, in contrast to others (whether higher- or lowerlevel), is based on the plain host language. It demonstrates that distribution can be exploited using a conventional object-oriented language without extending the language proper and without intervening into available compilers and runtime systems.

At the same time, having used Eiffel as a model language of object-oriented concepts, the HERON system proved well prepared for its heterogeneous extension. The realization of the distribution system as a non-integrated, add-on system expansion bears some implementation problems, but the goal of a modular solution is a suitable basis for heterogeneous system extension and is worth the effort. Whenever transparent distribution is desired but not being offered by available compilation systems the approach presented here would be appropriate.

\section{REFERENCES}

[ACHAuer 1993] B. Achauer: The Dowl distributed object-oriented language. Communications of the ACM 36, 9.

[Andrews Olsson 1993] G.R. Andrews, R.A. Olsson: The SR Programming Language. Benjamin/ Cummings.

[Auerbach Russell 1994] Joshua S. Auerbach, James R. Russell: The Concert Signature Representation: IDL as Intermediate Language. ACM SIGPLAN Notices 29, 8 (August 1994).

[Bennet 1990] J.K. Bennet: Experience with Distributed Smalltalk. Software - Practice \& Experience 20, 2.

[Birrel Nelson i984] Andrew D. Birrell, Bruce Jay Nelson: Implementing Remote Procedure Calls. ACM Transactions on Computer Systems 2, 1, 39-59.

[BoRLAND 1993] Borland International Inc.: Borland Pascal mit Objekten 7.0. Borland GmbH Langen, München: text und form.

[FInke ET AL 1993] S. Finke, P. Jahn, O. Langmack, K.-P. Löhr, I. Piens, Th. Wolff: Distribution and Inheritance in the HERON Approach to Heterogeneous Computing. 13. Int. Conf. on Distrib. Computing Systems, Pittsburgh, IEEE.

[LöHr Piens Wolff 1994] Klaus-Peter Löhr, Irina Piens, Thomas Wolff: Verteilungstransparenz bei der objektorientierten Entwicklung verteilter Applikationen. OBJEKTspektrum November/ Dezember 1994 Nr 5. 
[Gunaseelan LeBlanc 1992] L. Gunaseelan, Richard J. LeBlanc Jr: Distributed Eiffel: A Language for Programming Multi-Granular Distributed Objects. Proc. 4th Int. Conf. on Computer Languages, IEEE.

[Herlihy Liskov 1982] M. Herlihy, B. Liskov: A Value Transmission Method for Abstract Data Types. ACM Transactions on Progr. Languages and Systems 4, 4, pp 527-551.

[Hoffmann Weinberger Wolff 1995] Peter Hoffmann, Anton Weinberger, Thomas Wolff: Heterogene Transparenz bei der Verteilung von Objekten in HERON. Workshop Anwendungsunterstützung für heterogene Rechnernetze, Freiberg März 1995.

[Horn Cahill 1991] C. Horn, V. Cahill: Supporting distributed applications in the Amadeus environment. Computer Communications 14 (June 1991).

[Johnson Leach 1987] Herrick J. Johnson, Paul Leach: Network Computing System. apollo White Paper, Apollo Computer Inc.

[Jones Rashid 1986] Michael B. Jones, Richard F. Rashid: Mach and Matchmaker: Kernel and language support for object-oriented distributed systems. Technical Report CMU-CS-87-150, CS Department, CMU.

[McHugh Cahill 1993] Colm McHugh, Vinny Cahill: Eiffel**: An Implementation of Eiffel on Amadeus, a Persistent, Distributed Applications Support Environment. Proc. TOOLS Europe '93, Prentice-Hall.

[MeYER 1992] Bertrand Meyer: Eiffel: The Language. Prentice Hall.

[Monninger 1993] Frieder Monninger: Eiffel - Objektorientiertes Programmieren in der Praxis. Heise, Hannover.

[MPI 1994] Message Passing Interface Forum: MPI: A Message-Passing Interface Standard. University of Tennessee, Knoxville, Computer Science Department Technical Report CS-94-230, May 1994 / International Journal of Supercomputing Applications 8, 3/4.

[Nolte 1994] Jörg Nolte: Duale Objekte - Ein Modell zur objektorientierten Konstruktion von Programmfamilien für massiv parallele Systeme. Dissertation, Freie Universität Berlin.

[OMG 1991] The Object Management Group (OMG): The Common Object Request Broker: Architecture and Specification (CORBA). OMG Document Number 91.12.1.

[OSF 1993] Open Software Foundation: DCE Application Development Reference. Prentice-Hall.

[PIENs 1993] Irina Piens: HERON - Konfiguration verteilter Eiffel-Programme. GI/ITGArbeitskreistreffen ,Entwicklung und Management verteilter Anwendungssysteme' Frankfurt/ Main, 14.-15. Oktober 1993.

[RAj ET AL 199I] R.K. Raj, E. Tempero, H.M. Levy, A.P. Black, N.C. Hutchinson, E. Jul: Emerald: a general-purpose programming language. Software - Practice and Experience 21, 1.

[SCHILl Mock 1993] Alexander B. Schill, Markus U. Mock: $D C++$ : distributed object-oriented system support on top of OSF DCE. Distrib. Syst. Engng 1, 112-125.

[Sunderam 1990] V.S. Sunderam: PVM: A framework for parallel distributed computing. Concurrency: Practice and Experience 2, 4, 315-339.

[WolfF 1988] Thomas Wolff: Organisation of mixed language programming in heterogeneous distributed systems. ESPRIT project Delta-4 report 4.5.

[WolfF 1995] Thomas Wolff: Transparent Object Distribution and Remote Inheritance. In Amr Zaky: Tools and Environments for Parallel and Distributed Systems. Kluwer (to appear). 


\section{BIOGRAPHY}

Klaus-Peter Löhr got his PhD in Computer Science from Technische Universität Berlin in 1974. After four years as an Assistant Professor at the department, working mainly in operating systems, he was appointed Associate Professor at Universität Bremen. From 1985 until now, he has been Professor at the Institute of Computer Science, Freie Universität Berlin, leading a working group on systems software and software engineering. The main research interests of Dr. Löhr are concurrent systems and distributed object systems.

Thomas Wolff studied Computer Science at Universität Kiel. His interests in programming languages and concurrency evolved to include distribution and object orientation at Freie Universität Berlin where he is currently approaching his $\mathrm{PhD}$. 